\title{
A Differential Electrochemical Readout ASIC with Heterogeneous Integration of Bio-nano Sensors for Amperometric Sensing
}

\author{
Sara S. Ghoreishizadeh*, IEEE Member, Irene Taurino, IEEE Member, Giovanni DeMicheli, IEEE Fellow, \\ Sandro Carrara, IEEE Fellow, Pantelis Georgiou, IEEE Senior Member \\ * s.ghoreishizadeh14@imperial.ac.uk
}

\begin{abstract}
A Monolithic biosensing platform is presented for miniaturized amperometric electrochemical sensing in CMOS. The system consists of a fully-integrated current readout circuit for differential current measurement as well as on-die sensors developed by growing Platinum nano-structures (Pt-nanoS) on top of electrodes implemented with the top metal layer. The circuit is based on the switch-capacitor technique and includes pseudodifferential integrators for concurrent sampling of the differential sensor currents. The circuit further includes a differential to single converter and a programmable gain amplifier prior to an ADC. The system is fabricated in $0.35 \mu \mathrm{m}$ technology and measures current within $\pm 20 \mu \mathrm{A}$ with minimum input-referred noise of $0.47 \mathrm{pA}$ and consumes $9.3 \mathrm{~mW}$ from a $3.3 \mathrm{~V}$ supply. Differential sensing for nano-structured sensors is proposed to build highly-sensitive and offset-free sensors for metabolite detection. This is successfully tested for bio-nano-sensors for the measurement of glucose in sub-mili Molar concentrations with the proposed readout IC. The on-die electrodes are nanostructured and cyclic voltammetry run successfully through the readout IC to demonstrate detection of $\mathrm{H}_{2} \mathrm{O}_{2}$.
\end{abstract}

Index Terms-Differential readout, Potentiostat, switchcapacitor, Metabolite sensing, Bio-nano-sensor, on-die sensor, Ptnanostructure, Glucose sensor

\section{INTRODUCTION}

$\mathbf{P}$ OINT-of-care diagnostics (POCD) allow faster test results and immediate clinical decisions to be made. Such testing requires biosensing platforms that are sensitive and selective to the target metabolites, fast, autonomous, and ideally inexpensive. Electrochemical biosensors are good candidates for POCD. They are commonly used to detect biomolecules in blood samples in clinical laboratories or at home, for example to monitor Diabetes by measuring glucose.

An array of such sensors where each pixel is made selecetive to a particular biomolecule (through the use of selective enzymes) enables a thorough study of different metabolites in human blood, interstitial or tear fluid [1], [2]. The integration of miniaturized biosensor arrays with readout instrumentation introduces the opportunity for a low-cost and massmanufacturable sensing platform. The integration also improves the signal to noise ratio (SNR) of the measurements and

S. S. Ghoreishizadeh and P. Georgiou are with the department of Electrical $\&$ Electronic Eng. and the Centre for Bio-Inspired technology at Imperial College London, UK.

I. Taurino was and S. Carrara and G. De Micheli are with the Integrated Systems Laboratory (LSI), École Polytechnique Fédérale de Lausanne (EPFL), Switzerland. I. Taurino is currently with Philip Morris International. provides scalability by removing the long wire interconnects. Such platforms have been introduced for DNA analysers using affinity-based sensors [3], [4].

A main challenge in enzymatic-sensor miniaturization is the detection of a small signal levels (in pico- or femtoampere range) due to small sensor area. Three-dimentional structures such as Carbon-nano-tubes (CNT) [5] or gold nanostructures [3] have been proposed where their integration to the sensor increases the current level as well as its sensitivity and results in a better Limit of Detection (LOD). Recently Pt nano-structures (Pt-nanoS) are introduced which also allow non-enzymatic detection of glucose [6].

Generally, the sensor current can be defined as the current that passes through the Working electrode (WE) of a threeelectrode based electrochemical cell when the sensor is stimulated by a fixed (e.g. in Chronoamperometry technique) or a triangular (e.g. in Cyclic Voltammetry technique) voltage waveform, among others. The sensor current, $I_{W E}$, consists of a background current and a redox current. The background current does not depend on the concentration of the target biomolecule and is partly due to the interferences from other electro-active molecules in the solution as well as to layering phenomena. Both components of the background current typically increase significantly in presence of nano-structures where in particular the capacitive current increases [7]. The presence of a large background current when reading out a small redox current requires a high resolution ADC and increases the power consumption of the readout circuit.

Differential measurement of electrochemical sensors is proposed to remove the background current from the measurements in one of the following ways $(i)$ to measure and store the background current in absence of the target biomolecule and subtract it from the sensor current that is measured in presence of the biomolecule [8], [9]. This method is effective when there is a rapid change in the concentration of the biomolecule such as in the case of dopamine. This is not the case for glucose, lactate and other metabolites with gradually changing concentration. (ii) to use an extra sensor $\left(\mathrm{WE}_{b k g}\right)$ to record the background current and subtract it from the main sensor $\left(\mathrm{WE}_{\text {main }}\right)$ current in real-time. Differential chargebased sensing using two electrodes has been implemented in [10] for DNA detection where the charges generated at the two electrodes, $\mathrm{WE}_{b k g}$ and $\mathrm{W} E_{\text {main }}$ are sampled at different phases of the clock. 
Based on the latter technique, in this paper we present a novel fully-integrated differential readout IC (DiRIC) for amperometric sensing to remove the background current produced by a $\mathrm{WE}_{b k g}$ from the current of $\mathrm{WE}_{\text {main }}$ in real-time. The $\mathrm{WE}_{b k g}$ is designed to be minimally sensitive to the target biomolecule so that the current difference still shows a high sensitivity. Such extra sensor is produced by designing $\mathrm{WE}_{b k g}$ and $\mathrm{WE}_{\text {main }}$ with a similar geometry and structure as will be discussed in this work. DiRIC is based on a chain of switchcapacitor (SC) circuits that sample both sensor currents at the same clock phase and removes the background current in analog domain. The system provides wide dynamic range in current detection and enables small current measurement in presence of potentially large background current.

The simulation results of DiRIC were first introduced in [11]. In this paper we advance this to show the fabricated system and an evaluation of its performance through electrochemical measurements performed with a variety of sensors. This is the first demonstration of differential measurement for amperometric sensors and is achieved through the codesign of the bio-nano-sensor and DiRIC. We propose a method to develop a $\mathrm{WE}_{b k g}$ to provide a current that resembles the background (or offset) current of $\mathrm{WE}_{\text {main }}$. Through glucose measurements with the developed bio-nano-sensors and DiRIC, we show that differential sensing is effective in reducing the offset current as well as the current drift.

We also demonstrate for the first time the heterogeneous integration of bio-nano-sensors with the readout system on CMOS by growing Pt-nanoS directly on the top metal layer. We show that the introduction of Pt-nanoS significantly increases the sensor current. Moreover, we present preliminary results for the detection of $\mathrm{H}_{2} \mathrm{O}_{2}$ with the developed platform.

This paper is organized as follows: in Section II the design of different circuit blocks in DiRIC is presented. In Section III the fabricated system as well as the electrical characterisation of DiRIC are shown. In Section IV we take the example of glucose sensor and propose a protocol to prepare $\mathrm{WE}_{b k g}$ and $\mathrm{WE}_{\text {main }}$, and present differential measurements acquired with DiRIC and the developed sensors. Section IV also includes the development of on-die sensors and measurements with the integrated sensing platform. The conclusions are drawn in Section V.

\section{Differential Readout IC (DiRIC)}

DiRIC consists of a potentiostat to control the voltage of the sensor and a differential readout circuit to measure the differential sensor current. The top level system architecture of DiRIC is shown in Fig. 1. DiRIC can readout both ondie and external (off-die) sensors to study different sensing configurations and readout various sizes and types of sensors. A typical electrochemical sensor consists of a reference electrode (RE) and a counter electrode (CE) in addition to the WE. A second WE, $\mathrm{WE}_{b k g}$, is added herein to the sensor to demonstrate differential sensing.

\section{A. Readout chain}

The current readout circuit is designed to measure the currents of $\mathrm{WE}_{\text {main }}$ and $\mathrm{WE}_{b k g}$ with two integrator trans-

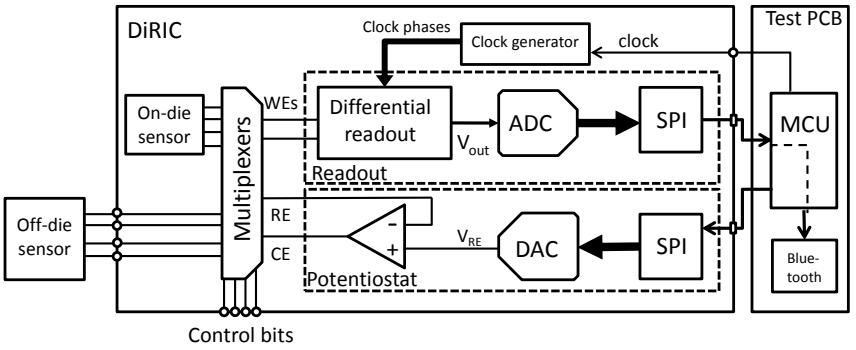

Fig. 1: The high level block diagram of DiRIC with connections to the test PCB and off-die sensors

impedance amplifiers. The difference of the currents is then amplified before the ADC. The schematic view of the readout chain is shown in Fig. 2 and consists of four main building blocks:

(i) Two 6-to-1 multiplexers select the sensors to be readout. The multiplexers are externally controlled through digital control bits and are implemented with transmission gates. Relatively big transistors $(\mathrm{w} / \mathrm{l}=5 / 0.35 \mu \mathrm{m})$ were used to reduce $r_{\text {on }}$ and ensure accurate voltage control of the sensors regardless of the sensor current.

The multiplexer at the main integrator connects the integrator to one of the three on-die $\mathrm{WE}_{\text {main }}$, two I/O pads or an ISFET. The I/O pads can either be connected to off-die WEs or be used to test the readout circuit. The multiplexer at the background integrator is connected to an on-die electrode $\left(\mathrm{WE}_{b k g}\right)$, a REFET and a MOSFET for differential $\mathrm{pH}$ sensing [11], as well as an I/O pad. Two dummy (i.e. always off) connections (to $V_{\text {dummy }}=V_{R E}$ ) are also included to equalise the parasitic capacitance at the input of the integrators as well as the leakage current of the two multiplexers. The latter is mainly due to the off-state leakage current of the switch transistors. The leakage equalisation is optimised for the on-die sensing mode where the voltage at the two disconnected on-die $\mathrm{WE}_{m}$ ains is assumed to be equal to $V_{R E}$. The connections of the multiplexers are shown in Fig. 2. DiRIC works in both Single-mode and differential-mode, where in single-mode, the multiplexer disconnects the reference integrator from any input.

(ii) Two identical integrators convert the sensor current into voltage. The three main benefits of using a SC-based integrator over a resistor-based TIA [5] and a current-mode potentiostat [12], [13], [14] are: The sensor current is low-pass filtered straight away through the integration, which removes the high frequency noise in the electrochemical process; The SC-based integrator can measure bidirectional input currents which is in particular required to perform a cyclic voltammetry measurement; Finally, currents within a wide dynamic range can be measured by tuning the reset clock of the integrator.

The sensor currents $I_{\text {main }}$ and $I_{b k g}$ are integrated during the Integration phase (when $\phi_{1}=0$ ). During the Reset phase (when $\phi_{1}=1$ ), $C_{i n t}$ discharges through $S_{L L}$. The low-leakage switch, $S_{L L}$, also enables the correct voltage on the WEs through the negative feedback as well as a path for the sensor current during the Reset phase. 


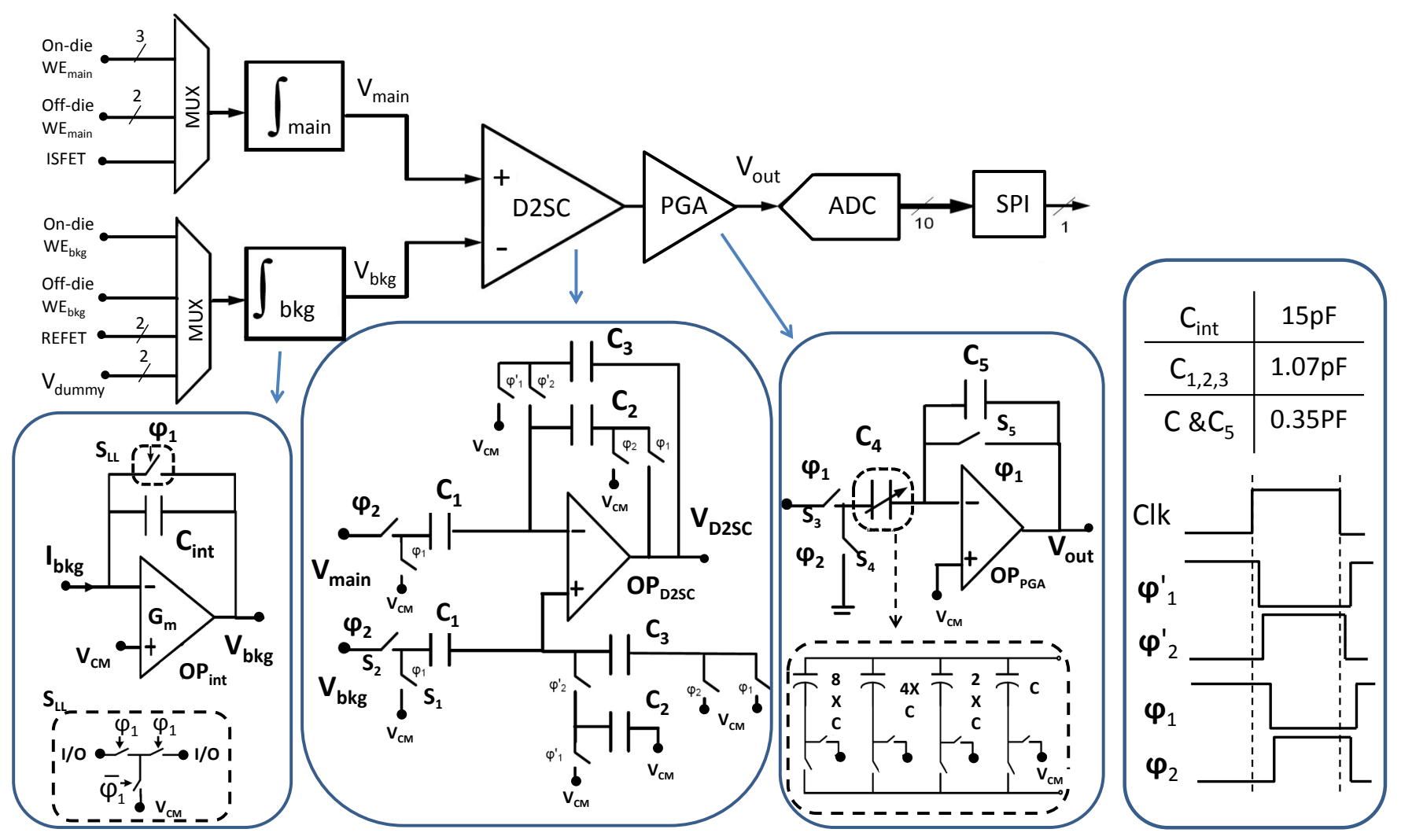

Fig. 2: The block diagram of readout circuit in DiRIC to measure and amplify the difference of two sensor currents.

The presented configuration of multiplexer and integrator ensures that the applied voltage to the sensor is well-controlled (i.e. fixed at $V_{C M}$ ) during the whole clock period, and there is always a path for the sensor current either through $C_{\text {int }}$ (during the Integration phase) or through $S_{L L}$ (during the Reset phase).

(iii) A differential to single converter (D2SC) takes the difference of $V_{\text {main }}$ and $V_{b k g}$. Here, the SC technique is chosen over a resistor-based difference amplifier, because the output of the integrator is valid at the end of the Integration phase and needs to be sampled right away. Moreover, the autozeroing technique can be easily implemented within a SCbased design to reduce the low frequency noise and cancel the opamp offset.

The SC-based D2SC is designed similar to [15]. It samples the two inputs at the same clock phase and uses correlated double sampling (CDS) to cancel the clock-feed-through and op-amp offset voltage. D2SC tracks $V_{\text {main }}$ and $V_{b k g}$ during the Integration phase and holds right before the Reset phase begins, when $\phi_{2}$ is inserted. $\phi_{1}$ and $\phi_{2}$ are non-overlapping clock cycles produced by the clock generator circuit. During the Integration phase $C_{3}$ is connected in feedback around $O P_{D 2 S C}$ and causes the output to change only by the opamp input offset voltage. This is a much smaller change than that required without the usage of $C_{3}$ where the output has to slew to a voltage close to $V_{C M}$ each time the amplifier is reset. The output of D2SC is valid during the Reset phase (i.e. $\phi_{1}=1$ ). To achieve a unity gain, $C_{1}, C_{2}$, and $C_{3}$ have similar values.

The clock waveforms with the primed superscripts change before the non-primed waveforms in order to prevent charge from escaping through $C_{3}$ and $C_{3}^{\prime}$. The $O P_{D 2 S C}$ is left openloop for this amount of time. However, this has no effect on the ultimate output of the D2SC circuit except for causing glitches at the clock transitions [15].

(iv) A programmable gain amplifier (PGA) amplifies the output of the differential SC amplifier. The PGA is realized in a $\mathrm{SC}$ architecture where the gain is proportional to the ratio of the capacitors $C_{4}$ and $C_{5}$ where $C_{4}$ is a programmable capacitor array with a unit capacitor equal to $C_{5}$ and four bit dynamic range. Therefore, the gain of the PGA can be programmed to any integer number from 1 to 16 .

The output stage includes a 10 bit-SAR ADC (from standard AMS library cell) and a serializer enabling voltages as small as $3.3 \mathrm{mV}$ to be resolved at the output of PGA.

\section{B. Potentiostat}

The RE and CE are controlled through an opamp in negative feedback, where different voltage profiles are generated using a 10-bit digital to analog converter (DAC, from AMS analog standard cell library). An off-chip microcontroller is programmed to drive the DAC through an SPI module. Different waveform profiles can be provided to control the cell in Cyclic voltammetry, Square-wave voltammetry and Chronoamperometry measurements.

\section{Clock generator}

All clock waveforms are generated on-chip by the clock generator circuit. The clock generator circuit is shown in Fig. 3 


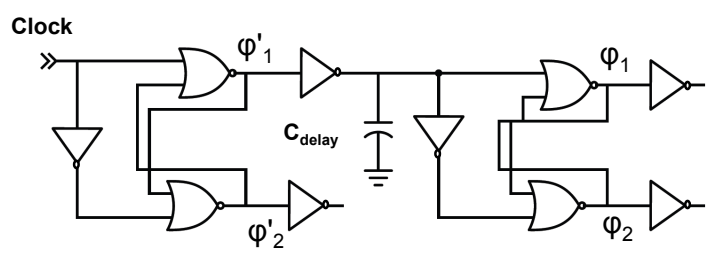

Fig. 3: The schematic of clock generator, adopted from [15].

where the inverted outputs were used to drive the PMOS transistors in the complementary switches. The clock waveforms with the primed superscripts change before the non-primed waveforms in order to prevent charge from escaping through $C_{3}$ and $C_{3}^{\prime}$ in the D2SC circuit. The actual amount of the delay between the clock phases and their primed version is determined by the capacitor $C_{\text {delay }}$ and is nominally $10 \mathrm{~ns}$ for $C_{\text {delay }}=0.5 \mathrm{pF}$.

\section{Amplifiers}

Single-stage amplifier is chosen for the integrators and the potentiostat to ensure the stability of $O P_{i n t}$ for a wide range of load capacitance (i.e. the sensor). Single-stage amplifier is also chosen for the PGA and D2SC over two-stage amplifiers to ensure short settling-time of the amplifier and potentially low power consumption. The folded-cascode structure is chosen due to its higher gain among single-stage amplifiers. To decrease the noise and the mismatch of the amplifiers, large PMOS transistors are used for the input pair as well as for the current mirror. The architecture of the PMOS-input folded-cascode amplifier with transistor dimensions designed for $O P_{\text {int }}$ is shown in Fig. 4.

The opamps in the integrator, D2SC, PGA have different output drive strength (and thus bias current). The potentiostat has to provide twice the current of the integrator to the load (i.e. the $\mathrm{CE}$ ), to accommodate for both $\mathrm{WE}_{b k g}$ and $\mathrm{WE}_{\text {main }}$ currents. Instead the opamp in the PGA should only satisfy the speed and slew-rate requirement where the PGA should settle within half of the fastest clock period. The simulated specifications of the opamps are listed in Table I. Monte-carlo simulation (with 200 samples, considering both mismatch and process variations) is used to find the standard deviation of the input offset voltage of the amplifiers.

\section{E. Readout gain}

The output voltage of the PGA, $V_{\text {out }}$, at the $n_{t h}$ clock cycle (where $\mathrm{n}$ is any integer number) after the start-up of DiRIC is calculated as a function of the input currents:

$$
\begin{aligned}
V_{\text {out }}(n T+3 T / 2)= & \frac{C_{4}}{C_{5}} \times V_{\text {out }, D 2 S C}(n T+T) \\
& =\frac{C_{4}}{C_{5}} \times \frac{T\left(I_{\text {main }}-I_{b k g}\right)}{2 C_{\text {int }}}
\end{aligned}
$$

where $T_{c l k}$ is the period of the clock. The transfer function of DiRIC is:

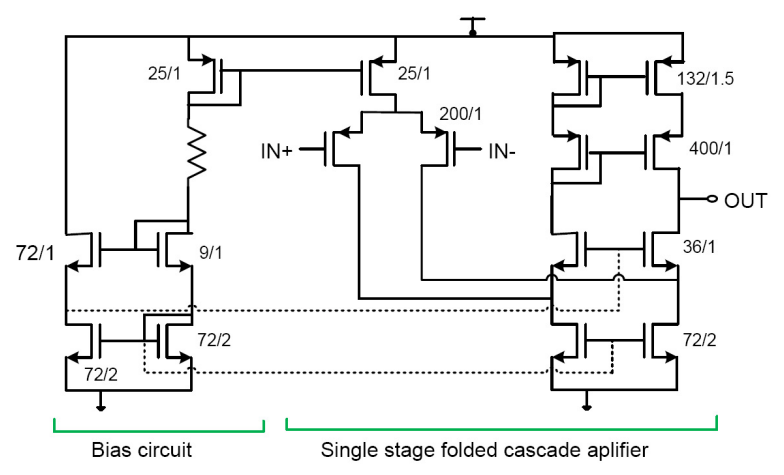

Fig. 4: The schematic of the single-stage folded-cascode amplifier used in the integrator, D2SC, PGA, and the potentiostat. The given transistor sizes are valid for the opamps used in the integrators.

TABLE I: Simulated specifications of the amplifiers in different circuits

\begin{tabular}{cccc}
\hline Parameter & integ, D2SC & PGA & ptnstat \\
\hline \hline Gain $(\mathrm{dB})$ & 82.7 & 83.5 & 71.5 \\
-3dB bandwidth $(\mathrm{kHz})$ & 8.4 & 4.3 & 45 \\
Phase margin $\left(\mathrm{C}_{L}=1 \mathrm{pF}\right)$ & $53^{\circ}$ & $60^{\circ}$ & $59^{\circ}$ \\
Power cons. $(\mathrm{mW})$ & 1.67 & 0.46 & 2.18 \\
Max output current $(\mu A)$ & 126 & 35 & 207 \\
Offset $(\mathrm{mV})$ & 1.64 & 1.63 & 1.65 \\
CMRR $(\mathrm{dB})$ & 126.7 & 124 & 112 \\
PSRR $(\mathrm{dB})$ & 84.4 & 84.6 & 72.5 \\
$\left(\mu \mathrm{V}, 1 \mathrm{mHz}-f_{-3 d B}\right)$ & 11 & 14 & 14.2 \\
\hline
\end{tabular}

$$
\frac{V_{\text {out }}(f)}{I_{d i f f}(f)}=\frac{C_{4}}{C_{5}} e^{-j 2 \pi f T_{c l k}} \frac{1-e^{\frac{-j 2 \pi f T_{c l k}}{2}}}{j 2 \pi f C_{\text {int }}}
$$

where $I_{d i f f}$ is defined as $I_{\text {main }}-I_{b k g}$. The transfer function is plotted in Fig. 5 for a clock frequency of $2 \mathrm{kHz}, C_{4} / C_{5}=1$, and $C_{i n t}=15 \mathrm{pF}$. The $f_{-10 d B}$ is defined as the frequency at which the gain drops by $10 \mathrm{~dB}$. The $f_{-10 d B}$ is considered the noise bandwidth of the system for noise calculations in the next section.

1) Gain error: The integrator is the main gain stage of the readout circuit and any non-ideality in the $S_{l l}$ or $O P_{i n t}$ affects the system gain. The sources of gain error are $(i)$ voltage drop across $S_{l l}$ during the Reset phase. This is due to the the passage of $I_{\text {main }}$ through the non-zero on-state resistance, $r_{o n}$, of $S_{L L}$ that prevents the full discharge of $C_{i n t}$ during the Reset phase. (ii) finite transconductance, $G_{m}$ of $O P_{i n t}$ which causes the input differential voltage of $O P_{\text {int }}$ to grow as the sensor current increases. The DC gain of the readout circuit is re-written considering the two error terms mentioned above:

$$
\frac{V_{\text {out }}}{I_{\text {in }}}=\frac{C_{4}}{C_{5}}\left(\frac{1}{2 f_{c l k} C_{\text {int }}}+r_{o n, S_{L L}}-\frac{1}{G_{m, O P_{\text {int }}}}\right)
$$

The main parameters of the integrator are summarized in Table III and are used to drive the gain as well as the gain error. 


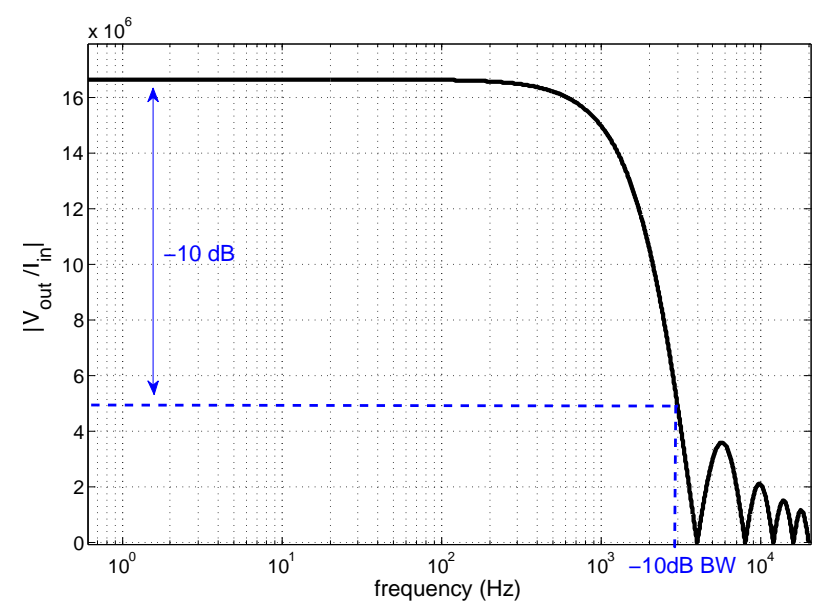

Fig. 5: The simulated transfer function of DiRIC for a $f_{c l k}=$ $2 k H z$. The $-10 \mathrm{~dB}$ bandwidth, $f_{-10 d B}$, is taken as the noise bandwidth.

\section{F. Noise}

The D2SC and PGA circuits use CDS technique to remove the opamp offset and reduce the $1 / \mathrm{f}$ noise. However, the inputreferred noise of $O P_{i n t}$ as well as the thermal noise due to $r_{o n, S_{L L}}$ couple directly to the integrator's output during the Integration phase, without being sampled. The integrator noise is then tracked and held by the D2SC and PGA where the foldover components are also added to it [16]:

$$
\begin{aligned}
& V_{n, \text { out }}^{2} \simeq\left(\frac{C_{4}}{C_{5}}\right)^{2} \times\left(V_{n, \text { op int }}^{2}+4 K T r_{\text {on }, S_{L L}}+\right. \\
& \left.\quad \quad \text { oldovers }\left.\left(V_{n, \text { op }_{\text {int }}}^{2}+4 K T r_{\text {on }, S_{L L}}\right)\right|_{f_{\text {clk }}}\right)
\end{aligned}
$$

where $\mathrm{T}$ is absolute temperature and $\mathrm{K}$ is the Boltzmann constant.

\section{FABRICATED SYSTEM}

DiRIC is fabricated in $0.35 \mu \mathrm{m}$ technology with two poly and four metal layers. The micro-photograph of the fabricated IC is shown in Fig. 6. The top metal layer is used to form the on-die sensor where a pad opening layer is used to remove the passivation layer and make the last metal layer exposed for post-processing. DiRIC occupies $0.6 \mathrm{~mm}^{2}$ and consumes an average power of $9.3 \mathrm{~mW}$ from a $3.3 \mathrm{~V}$ supply. From this the data converters (ADC and DAC) consume up to $1.1 \mathrm{~mW}$ and the breakdown on other circuits are listed in Table I. The on-die sensor measures $0.7 \mathrm{~mm} \times 0.4 \mathrm{~mm}$. The electrodes are placed in the middle of the chip to ensure more than $0.5 \mathrm{~mm}$ distance from the edges of the fabricated IC required for proper encapsulation of the chip and opening in the sensor area.

\section{A. Electrical measurement}

A PCB was made to test DiRIC in different modes and measurement techniques. An Atmel ATtiny 1634 micro-controller was embedded onto the test PCB as well as a Bluetooth chip. The microcontroller is programmed to drive the DAC (through SPI communication) in order to provide different voltage

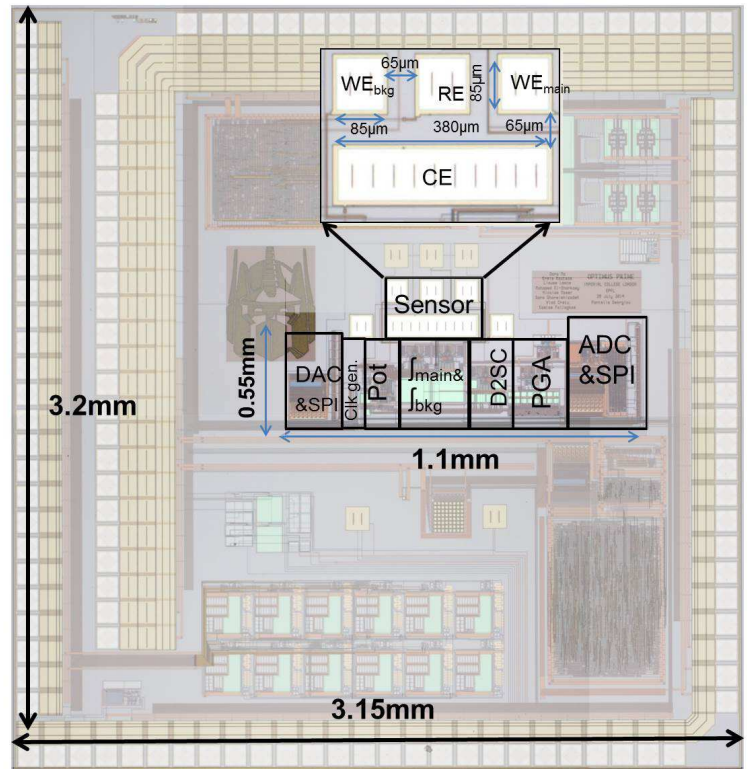

Fig. 6: The micro-photograph of integrated system where the on-die sensor array is highlighted. The array includes $\mathrm{WE}_{\text {main }}$, $\mathrm{WE}_{b k g}$, RE and CE.

waveforems (triangular, fixed, etc) on RE for the chosen measurement technique. The microcontroller also provides the clock, $f_{c l k}$, for DiRIC and reads the ADC through SPI. It then transmits ADC data via UART to the Bluetooth chip on the PCB which sends it to an Android tablet [17] where it is displayed and stored. The node $V_{\text {out }}$ is also buffered (through a $O P_{b u f f}$ which is similar in structure to $O P_{P G A}$ ) and accessible through a test pin. The clock frequency, $f_{c l k}$ is selected based on the maximum input current while the PGA is adjusted to amplify the input current difference.

A Keithley 2602 is used to provide a sink or source input current into DiRIC. Fig. 7 shows the $I_{i n}-V_{\text {out }}$ characteristics of DiRIC for different input current ranges and clock frequencies and in the single operation modes. The DC gain at each $f_{c l k}$ is reported in Table III. The single-mode operation shows excellent linearity at sub $10 \mu A$ range. The simulations show that the readout is linear for input currents up to $\pm 100 \mu \mathrm{A}$. However, this could not be measured due to slew-rate limitations of $O P_{b u f f}$ and speed limitation of the on-chip ADC.

The differential-mode is tested by fixing the difference current, $I_{\text {diff }}$, to different values from $10 n A, 100 n A$ and $1 \mu A$, and changing the background current, $I_{b k g}$ where $I_{\text {main }}=I_{b k g}+I_{d i f f}$. The frequency of the clock is chosen based on the maximum $I_{\text {main }}$ and the $C_{4} / C_{5}$ is chosen according to $I_{\text {diff }}$. The results are shown in Fig. 8 demonstrating the current subtraction accuracy of DiRIC for $I_{b k g}$ up to 40 times higher than $I_{d i f f}$. This is limited by getting close to the saturation of $O P_{i n t}$ for high $I_{b k g} / I_{d i f f}$ ratios. Therefore, the range can be further increased by increasing $f_{c l k}$ and $C_{4} / C_{5}$ ratios as is seen in the case when $I_{d i f f}=100 n A$.

The cyclic-voltammetry measurement was emulated by connecting a resistor $(3.3 M \Omega)$ between external RE and WE pins (when RE and CE pins are short connected, $f_{c l k}=7 \mathrm{kHz}$ 


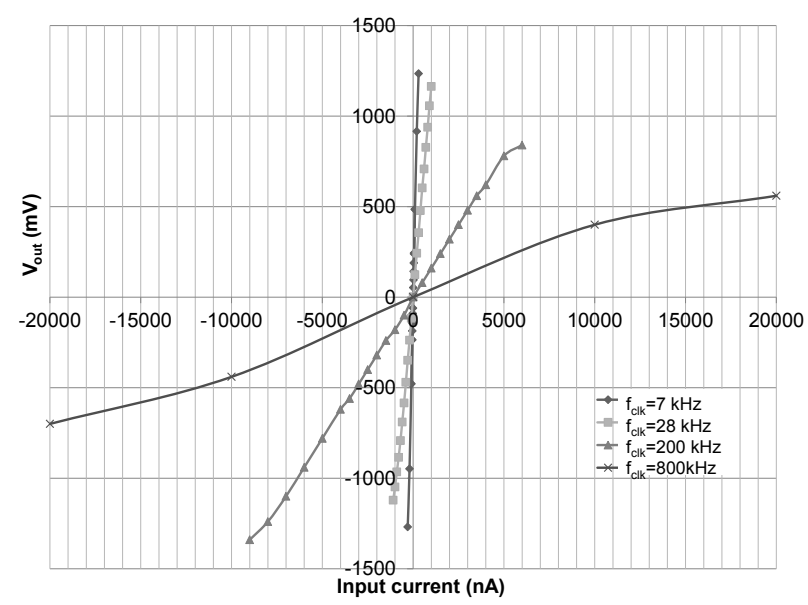

Fig. 7: The input-output characteristics of DiRIC for different clock frequencies $\left(f_{c l k}=7,28,200,800 \mathrm{kHz}\right.$ and $\left.C_{4} / C_{5}=1\right)$ in single mode operation.

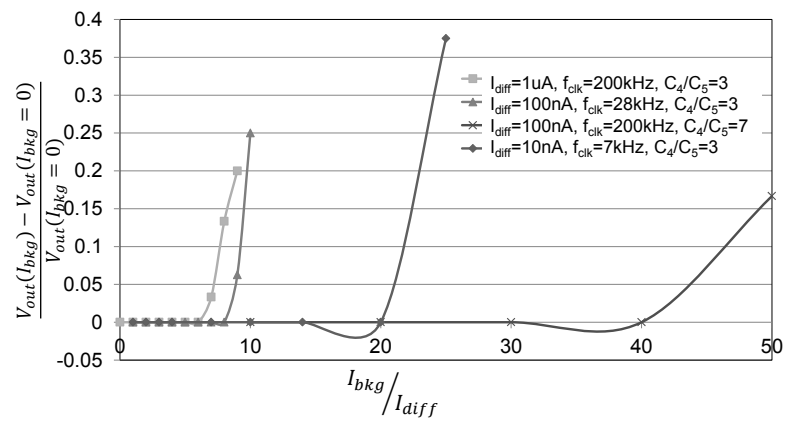

Fig. 8: The measured relative change in the output voltage versus the ratio of $I_{b k g}$ and $I_{d i f f}$, when $I_{\text {main }}=I_{b k g}+I_{d i f f}$. DiRIC is configured in the differential mode, $f_{c l k}$ is chosen according to maximum $I_{\text {main }}$.

and $C_{4} / C_{5}=1$ ). The microcontroller was programmed to drive the potentiostat to produce a triangular voltage on $\mathrm{RE}$ (See Fig. 9-(a)). The output of the ADC is stored on Android tablet and plotted versus time in Fig. 9 showing successful implementation of cyclic voltammetry. The input current is calculated from the measured waveforms across the resistor. The ADC output versus the input current to WE is plotted in Fig. 9-(b)) showing a linear response within the $\mathrm{nA}$ range.

1) Noise and leakage current: The noise at $V_{\text {out }}$ at different $f_{c l k}$ was measured at zero input current using a low-noise pre-amplifier (with $-3 \mathrm{~dB}$ bandwidth at $1 \mathrm{MHz}$ ) followed by a PicoScope 2205. Then from this, the total input-referred current noise, $I_{n, i n, t o t}$, is calculated by dividing the output noise, $V_{n, \text { out }}^{2}(f)$, by the absolute value of the transfer function and integration over the $-10 \mathrm{~dB}$ bandwidth, $f_{-10 d B}$, of the readout circuit.

$$
\begin{array}{r}
I_{n, \text { in }}^{2}(f)=\frac{V_{n, \text { out }}^{2}(f)}{\left|\frac{V_{\text {out }}(f)}{I_{\text {in }}(f)}\right|^{2}} \\
I_{n, \text { in }, \text { tot }}=\left(\int_{f_{0}}^{f_{-10 d B}} I_{n, \text { in }}^{2}(f) d f\right)^{1 / 2}
\end{array}
$$

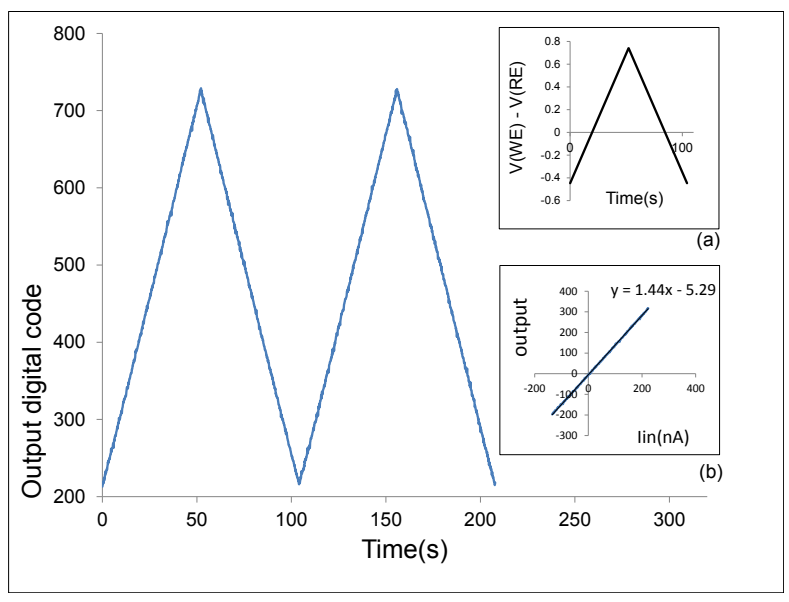

Fig. 9: The cyclic-voltammetry measurement emulated by connecting a $3.3 M \Omega$ resistor between RE and WE pins of DiRIC (where RE and CE pins are short connected). (a) the applied voltage across the resistor. (b) The Iin-Vout characteristics of DiRIC extracted from this measurement.

where $f_{0}$ is the minimum frequency in noise measurement and is listed in Table II for different $f_{c l k}$.

The integrated input-referred current noise, $I_{n, i n, t o t}$ versus $f_{c l k}$ is plotted in Fig. 10 and summarized in Table II. $I_{n, i n, t o t}$ decreases by decreasing $f_{c l k}$, and is as low as $0.47 \mathrm{pA}$ at $f_{c l k}=20 \mathrm{~Hz}$. The linear trend can be explained in the following way. (i) When $f_{c l k}$ is higher than the bandwidth of $O P_{\text {int }}\left(f_{-3 d B, O P_{\text {int }}}, 8.3 \mathrm{kHz}\right.$ according to Table I), By reducing $f_{c l k}$ by a factor of ten, the DC gain, $\frac{V_{\text {out }}}{I_{\text {in }}}$ increases and $f_{-10 d B}$ decreases ten times. However, the fold-over components in $V_{n, \text { out }}^{2}$ (Eqn 6) also increase by almost the same factor. Therefore, the integrated $I_{n, i n}$ within the $f_{-10 d B}$ bandwidth decreases by almost a factor of ten. (ii) When $f_{c l k}$ is higher than $f_{-3 d B, O P_{i n t}}$, the fold-over components are negligible and remain so by increasing $f_{c l k}$. Similarly, the content of the input-referred current noise at frequencies between $f_{-3 d B, O P_{\text {int }}}$ and $f_{-10 d B}$ is negligible. Therefore by increasing $f_{c l k}$ by an order of ten, $I_{n, i n, t o t}$ increases almost ten times. The leakage current of the switches in the multiplexer introduce an offset in the measurements and can saturate $O P_{\text {int }}$ at low $f_{c l k}$. The measured input leakage current is $2.1 \mathrm{nA}$ and was removed from plots in Figs. 7, 8, and 18-(b). This leakage current saturates the readout circuit when $f_{c l k}$ is smaller than $20 \mathrm{~Hz}$. The measured leakage current on three ICs was 2.1, 2.95 and $3 \mathrm{nA}$ and is reported in Table III along with other specifications of the readout circuit.

Using DiRIC, the minimum detectable change in sensor current is $0.47 p A$ while the maximum measurable current is $20 \mu \mathrm{A}$. This leads to a large cross-scale dynamic range of $156 \mathrm{~dB}$. A comparison with state-of-the-art will be presented in the next section.

\section{ELECTROCHEMICAL MEASUREMENT}

DiRIC can couple to and readout a variety of sensors thanks to its high input dynamic range and its support of different measurement modes and techniques. The versatility of DiRIC 


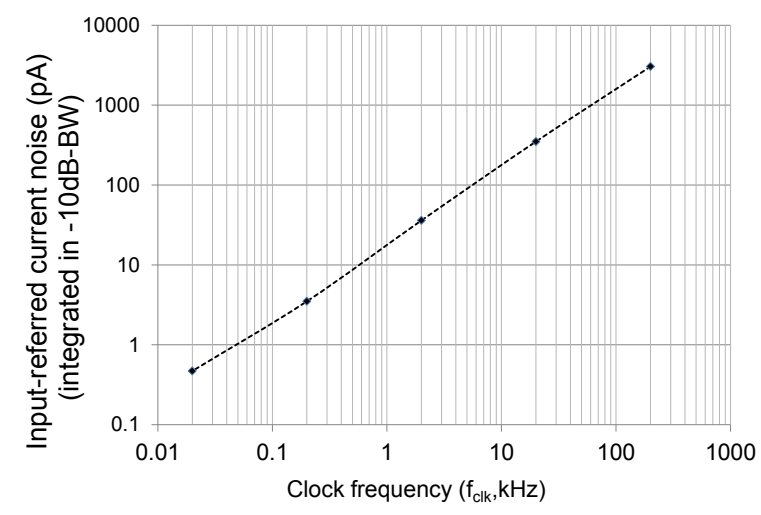

Fig. 10: The input referred current noise at different system clock frequencies, integrated within a bandwidth of $-10 \mathrm{~dB}$

TABLE II: Integrated input-referred current noise and parameters

\begin{tabular}{ccccc}
\hline$f_{c l k}(\mathrm{kHz})$ & $f_{0}(\mathrm{~Hz})$ & $f_{-10 d B}(\mathrm{kHz})$ & $\left.\frac{V_{\text {out }}}{I_{\text {in }}}\right|_{D C}(M \Omega)$ & $I_{n, \text { in }, \text { tot }}$ \\
\hline \hline 200 & 30.5 & 295 & 0.167 & $3.04 \mathrm{nA}$ \\
20 & 3 & 29.5 & 1.667 & $0.35 \mathrm{nA}$ \\
2 & 0.6 & 2.95 & 16.67 & $36 \mathrm{pA}$ \\
0.2 & 0.06 & 0.295 & 166.7 & $3.5 \mathrm{pA}$ \\
0.02 & 0.015 & 0.029 & 1667 & $0.47 \mathrm{pA}$ \\
\hline
\end{tabular}

has been demonstrated in this section through measurements with a range of on-die and off-die sensors. We propose and test a protocol to prepare $\mathrm{WE}_{b k g}$ and $\mathrm{WE}_{\text {main }}$ for differential sensing of glucose. We also demonstrate for the first time the heterogeneous integration of Pt-nanoS on CMOS to develop integrated platform for amperometric electrochemical sensing.

\section{A. Off-die sensors}

1) Differential sensor preparation: Screen printed electrodes (SPE) with one CE, one RE and two WEs were purchased from Dropsens. Pt-nanoS was electrodeposited onto both WEs from a solutions containing $50 \mathrm{mM} \mathrm{H}_{2} \mathrm{SO}_{4}$ (95$98 \%$, Sigma) and $25 \mathrm{mM} \mathrm{H}_{2} \mathrm{PtCl}_{6}$ (Aldrich) using a commercial potentiostat to perform Linear Scan Voltammetry (LSV) between $0 \mathrm{~V}$ and $1 \mathrm{~V}$ with respect to an $\mathrm{Ag}$ reference electrode. Here, a Pt electrode with an area of $12.54 \mathrm{~mm}^{2}$ was used as CE and placed in parallel to WE of the SPE. After the deposition process, a material activation was carried out by acquiring multiple cyclic voltammograms between $-0.2 \mathrm{~V}$ and $+1.5 \mathrm{~V}$ at $100 \mathrm{mV} / \mathrm{s}$ in $0.1 \mathrm{M} \mathrm{H}_{2} \mathrm{SO}_{4}$ until the overlap of two subsequent voltammograms was observed.

The morphology of the obtained nanostructures on the WEs was observed using a scanning electron microscope (SEM) image. The SEM image shows a very high density of Pt-nanoS was obtained (Fig. 11-right).

To prepare $\mathrm{WE}_{\text {main }}$ one of the Pt-nanostructurd WE in the SPE was functionalized with a $5 \mu \mathrm{l}$ drop consisting of glucose oxidase (GOD) and the cross-linker, Glutaraldehyde (GA) (both from Sigma-Aldrich, $15 \mathrm{mg} / \mathrm{ml} \mathrm{GOD}$ in $100 \mathrm{mM}$ PBS with GA $2.5 \% v / v$ as cross-linker in 9:1 volume ratio).
TABLE III: IC characteristic

\begin{tabular}{cc}
\hline Parameter & Value \\
\hline \hline Gain settings $(\mathrm{GS})$ & $1,2,3,4$ \\
$f_{\text {clk }}(\mathrm{kHz})(\mathrm{GS} 1 \mathrm{to} 4)$ & $7,28,200,800$ \\
DC Gain $(\mathrm{V} / \mu \mathrm{A})(\mathrm{GS} 1$ to 4$)$ & $4.4,15,0.16,0.042$ \\
Input current range $(\mathrm{GS} 1$ to 4$)$ & $\pm 0.3, \pm 1, \pm 6, \pm 20 \mu \mathrm{A}$ \\
\hline Measurement modes & single-input; differential-input \\
Measurement techniques & Chronoamperometry; Cyclic voltammetry \\
\hline Max measurable current & $20 \mu \mathrm{A}$ at $f_{c l k}=800 \mathrm{kHz}$ \\
Min total input-referred-noise & $0.47 \mathrm{pA}$ at $f_{\text {clk }}=20 \mathrm{~Hz}$ \\
Input leakage current & $(2.5 \pm 0.45) \mathrm{nA}$ on three ICs \\
\hline$G_{m, O P_{i n t}}$ & $1.8 \mathrm{~mA} / \mathrm{V}$ \\
$r_{\text {on, } S_{L L}}$ & $1.2 \mathrm{k} \Omega$ \\
$C_{\text {int }}$ & $15 \mathrm{pF}$ \\
\hline Technology & $0.35 \mu \mathrm{m}$ \\
Supply voltage & $3.3 \mathrm{~V}$ \\
Power consumption & $9.3 \mathrm{~mW}$ \\
Area & $0.6 \mathrm{~mm}$ \\
\hline
\end{tabular}
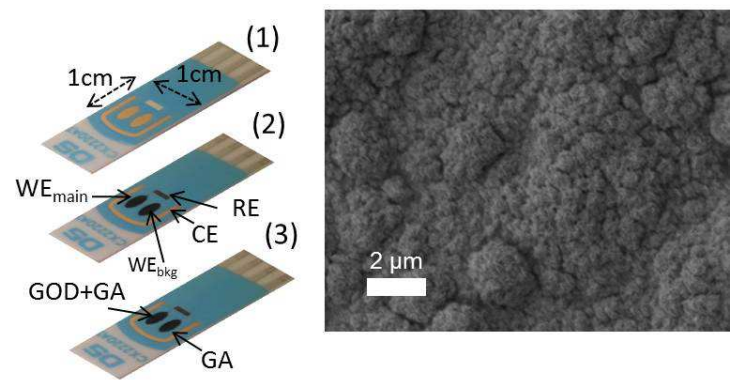

Fig. 11: Preparation steps of a dual-SPE for differential measurement of glucose: (1) The bare dual-SPE with bare gold electrodes; (2) Pt-nanoS electrodeposited on both $\mathrm{WE}_{\text {main }}$ and $\mathrm{WE}_{b k g}$; (3) GOD and the cross-linker Glutaraldehyde (GA) deposited on Pt-nanoS grown on $\mathrm{WE}_{\text {main }}$, while only GA is deposited on Pt-nanoS grown on $\mathrm{WE}_{b k g}$. The SEM image of the WE covered in Pt-nanoS in step two (right).

To form the $\mathrm{WE}_{b k g}$, a $5 \mu \mathrm{l}$ drop of GA $(2.5 \% v / v)$ was deposited on the other WE. This is to ensure $\mathrm{WE}_{b k g}$ has the closest surface structure to $\mathrm{WE}_{b k g}$ while it is still insensitive to glucose. The developed bio-nano-sensor is then kept at $4{ }^{\circ} \mathrm{C}$ overnight before use. The preparation steps of the electrodes are summarized in Fig. 11.

2) Effect of Pt-nanS on glucose sensor current: To demonstrate the effect of Pt-nanoS on the sensor sensitivity, a Chronoamperometry measurement for the detection of glucose is performed and results are shown in Fig. 12 using an SPE (as shown in Fig 11-step(1)). Here, one WE of the SPE is nanostructured with Pt-nanoS and functionalized with GOD and GA, while another WE was only functionalized with GOD and GA without being nanostructured. The measurements were done using a commercial potentiostat at different times on the same day by consecutively adding glucose by steps of $15 \mu M$ or $2.5 \mathrm{mM}$ to the PBS solution (Sigma, $100 \mathrm{mM}, \mathrm{pH}$ 7.4) under stirring and aerobic condition.

The calibration curves reported in Fig. 12 show that by introducing the Pt-nanoS the sensitivity (i.e. the slope of the calibration line) of the sensor increases by almost two orders 


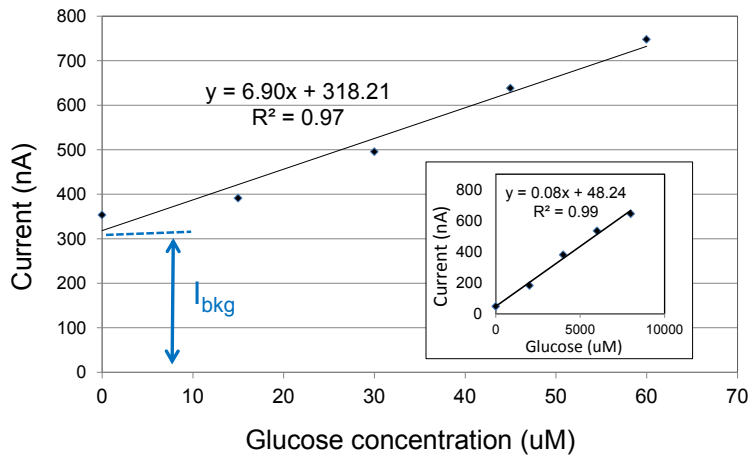

Fig. 12: Calibration curve measured during the chronoamperometry with glucose sensors enhanced by Pt-nanoS. Inner graph: Similar measurement without Pt-nanoS.

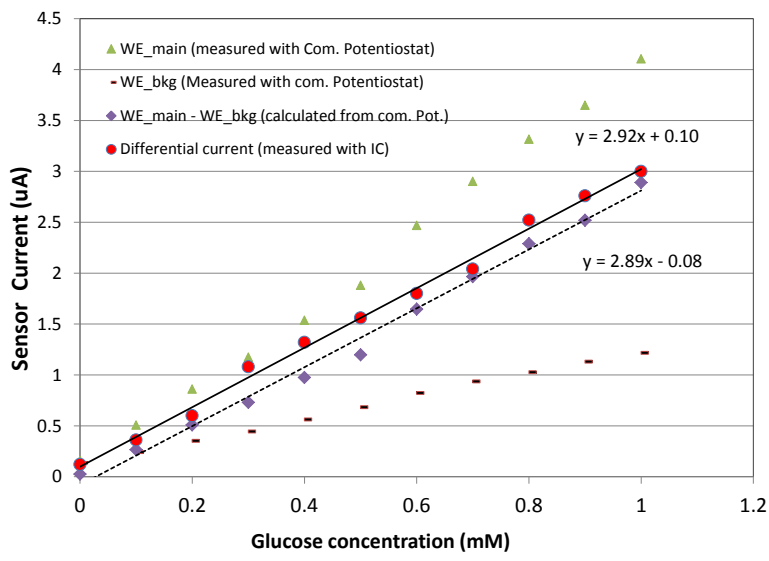

Fig. 13: The chronoamperometry of glucose using the dualSPE prepared according to Fig.11.

of magnitude (See Table IV). The background current $\left(I_{b k g}\right)$ of the sensor has also increased considerably.

3) Differential sensing of glucose: Chronoamperometry measurements of glucose were carried out at $+650 \mathrm{mV}$ in PBS solution (Sigma, $100 \mathrm{mM}, \mathrm{pH}$ 7.4) under stirring and aerobic condition by consecutively adding glucose (Sigma) up to $1 m M$ by steps of $100 \mu M$. The $\mathrm{WE}_{\text {main }}$ and $\mathrm{WE}_{b k g}$ were prepared according to the procedure shown in Fig. 11.

The sensors were conditioned for 50 minutes in the PBS solution before the first addition step. The current of $\mathrm{WE}_{\text {main }}$ and $\mathrm{WE}_{b k g}$ were measured simultaneously using a commercial potentiostat with two channels. The difference in the $\mathrm{WE}_{\text {main }}$ and $\mathrm{WE}_{b k g}$ currents was calculated after the measurements and plotted in Fig. 13.

The measurement was repeated on the same sensors and same day using DiRIC ( $\left.f_{c l k}=200 \mathrm{kHz}, C_{4} / C_{5}=1\right)$ to control and readout all four electrodes of the sensor. The results are plotted in Fig. 13 showing perfect matching of the two measurements as the slope of the differential calibration curves acquired by DiRIC is within $99 \%$ of the one measured with commercial potentiostat. The discrepancy in the differential offset between the two differential measurements can be due to the slight change of the offset current of either $\mathrm{WE}_{\text {main }}$ or $\mathrm{W}_{b k g}$ from one measurement to the other.
4) Discussions: Ideally, $I_{b k g}$ that is measured from $\mathrm{WE}_{b k g}$ does not change with glucose concentration. However, as seen from Fig. 13, $I_{b k g}$ changes with concentration. This is mainly due to the interference between the two WEs when the $\mathrm{H}_{2} \mathrm{O}_{2}$ generated at the vicinity of $\mathrm{WE}_{\text {main }}$ diffuses towards $\mathrm{WE}_{b k g}$ where it is oxidised and results in a current. The interference between the two electrodes can be defined as ratio of sensitivities of $\mathrm{WE}_{b k g}$ and $\mathrm{WE}_{\text {main }}$ to glucose:

$$
\text { Intereference }=\frac{S_{W E_{b k g}}}{S_{W E_{\text {main }}}}
$$

The interference is in theory independent from the sensitivity of the main sensor, $S_{W E_{\text {main }}}$ as the $I_{b k g}$ is a linear function of $I_{\text {main }}$. To minimise the interference, the stirring direction in above measurements were chosen such that the liquid moves away from the $\mathrm{WE}_{\text {main }}$ in an opposite direction to $\mathrm{WE}_{b k g}$.

The flow direction can be better controlled using a flowcell to decrease the interference. A measurement set-up with a flow-cell is sketched in Fig. 14 where the liquid flow is controlled to be in parallel with the WEs. Fig. 15 shows chronoamperometry measurement with a differential sensor using a flow rate of $25 \mu \mathrm{L} / \mathrm{min}$ and concentration steps of $1 \mathrm{mM}$. The differential sensor was prepared with the exact same procedure described in this section except that an aged GOD with a reduced activity was used which is expected to have less sensitivity and higher background current. The use of an aged enzyme was also thought to mimic the sensor operation towards the end of its lifetime.

Table IV summarizes the results with off-die glucose sensors. The observed interference measured using the flow-cell technique is as low as $20 \%$. We observed that the interference depends on the geometry of the flow cell, its alignment to the horizon and the flow rate. The interference may be minimized by making a seperating wall between the $\mathrm{WE}_{\text {main }}$ and $\mathrm{WE}_{b k g}$ as shown in other works [18]. Another way is to place the $\mathrm{WE}_{\text {main }}$ and $\mathrm{WE}_{b k g}$ further apart.

The measured sensor currents in Fig. 15 also shows the sensor current drifts in time at a fixed concentration of glucose. The drift is as high as $110 \mathrm{nA} / \mathrm{sec}$ in the highlighted time period in Fig. 15 and has been reduced to $45 \mathrm{nA} / \mathrm{sec}$. The current drift depends in part to the measurement condition such as flow rate, direction, and change in Oxygen concentration which contribute to mass transport; and in part to the enzyme condition and activity which determines the kinetic of the sensor. The mass-transport component of the drift is compensated for using the proposed differential measurement technique. However, only a part of the kinetic-related drift is removed and that is through the effect of the interference. In other words, assuming that $20 \%$ of the reported $60 \%$ driftreduction is due to the interference effect, the contribution of the mass-transport component is $40 \%$ of the whole drift and the remaining $60 \%$ could be attributed to the kinetic of the sensor.

\section{B. On-die sensors}

To develop and test the on-die electrodes, the IC was wirebonded to a test PCB. The wires were then covered with epoxy 
TABLE IV: Summary of Chronoamperometry measurements with off-die glucose sensors

\begin{tabular}{cccccccc}
\hline Reference & sample condition & \multirow{2}{*}{ Pt-nanoS } & $\left.I_{b k g}\right|_{0 \mu M}$ & $\left.I_{\text {main }}\right|_{0 \mu M}$ & $S_{W E_{\text {main }}}$ & $\frac{S_{W E_{b k g}}}{S_{W E_{\text {main }}}}$ & time-drift $(\Delta I / \Delta t)$ reduction \\
\hline \hline Fig. 12 & Stirring & No & - & $302 \mathrm{nA}$ & $0.08 \mu \mathrm{A} / \mathrm{mM}$ & - & NA \\
Fig. 12 & Stirring & Yes & - & $47 \mathrm{nA}$ & $6.9 \mu \mathrm{A} / \mathrm{mM}$ & - & NA \\
Fig. 13 & Stirring & Yes & $134 \mathrm{nA}$ & $158 \mathrm{nA}$ & $4 \mu \mathrm{A} / \mathrm{mM}$ & $28 \%$ & NA \\
Fig. 15 & flow cell & Yes & $300 \mathrm{nA}$ & $318 \mathrm{nA}$ & $0.68 \mu \mathrm{A} / \mathrm{mM}$ & $20 \%$ & up to $60 \%$ \\
\hline
\end{tabular}

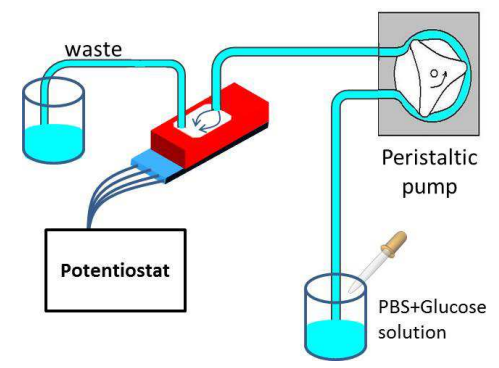

Fig. 14: The measurement set-up for the differential chronoamperometry of glucose using a flow cell

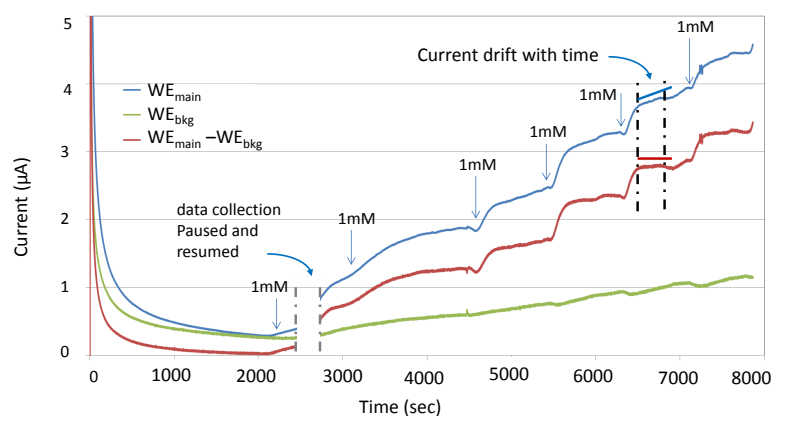

Fig. 15: The chronoamperometry for glucose measurement (with commercial potentiostat) with differential bio-nanosensor under the flow condition. Both drift and offset are reduced using differential measurement.

such that electrodes are exposed for post-processing.

1) Electrodeposition of Pt-nanoS: Pt-nanoS was deposited on the electrodes, that were made by the top metal layer, in the following way. An external $\mathrm{Ag} / \mathrm{AgCl}$ reference electrode and a Pt counter electrode were used as shown in Fig. 16. A drop of the solution (50 $\mathrm{mM} \mathrm{H}_{2} \mathrm{SO}_{4}(95-98 \%$, Sigma) and $25 \mathrm{mM}$ $\mathrm{H}_{2} \mathrm{PtCl}_{6}$ (Aldrich)) was placed to cover all the electrodes. Three repetitive LSVs from $0 V$ to $0.8 V$ were performed between the on-die WE versus the reference electrode using a commercial potentiostat.

The morphology of the WE surface was investigated with a Zeiss Merlin high resolution scanning microscopy after the measurements were performed. The SEM images are shown in Fig. 17 where the $\mathrm{Pt}$ nanoS are clearly visible.

2) Measurements with nanostructured on-die sensors: Measurements with on-die sensors were carried out before and after the addition of Pt-nanoS to the electrode. A drop of $100 \mathrm{mM}$ PBS solution containing 0 and $1 \mathrm{mM}$ concentrations of $\mathrm{H}_{2} \mathrm{O}_{2}$ was placed on top of the IC. The $\mathrm{Ag} / \mathrm{AgCl}$ reference

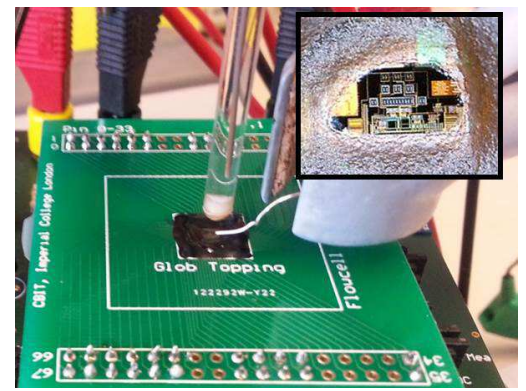

Fig. 16: The photograph of set-up for the electrodeposition of Pt-nanoS on on-die electrodes. $\mathrm{An} \mathrm{Ag} / \mathrm{AgCl}$ reference electrode and a $\mathrm{Pt}$ counter electrode were used to enhance the deposition. Inner image: the microphotograph of DiRIC and on-die electrodes inside the epoxy well.

electrode and the Pt counter electrodes were used. All three electrodes were connected to DiRIC for voltage control and current readout. The system clock, $f_{c l k}$, was set to $7 \mathrm{kHz}$ and a triangular waveform with the slope of $95 \mathrm{mV} / \mathrm{sec}$ was applied between $\mathrm{WE}_{\text {main }}$ and the reference electrode. The acquired three consecutive voltammograms are shown in Fig. 18 where the overlap of the voltammograms is the indication of the sensor stability.

Voltammograms measured on the same electrode before and after growing Pt-nanS are presented in Fig. 19. The peak-topeak sensor current from the four measurements in Fig. 19 are listed in Table $\mathrm{V}$ (under $\mathrm{WE}_{\text {mian,A }}$ ) showing an increase of more than one order of magnitude in the sensor current by introducing Pt-nanoS. There is also a threefold increase in the nanostructured sensor current in presence of $\mathrm{H}_{2} \mathrm{O}_{2}$. This demonstrates the high sensitivity of the sensor to $\mathrm{H}_{2} \mathrm{O}_{2}$ which is the main product of redox reaction in enzymatic electrochemical sensors such as glucose and lactate.

3) On-die sensor variability: The peak-to-peak current of the voltammograms acquired with an on-die Pt-nanoS $\mathrm{WE}_{\text {mian }}$ prepared as described in Section IV-B1 on a different IC is also reported in Table $\mathrm{V}$ (under $\mathrm{WE}_{\text {main }, B}$ ) for comparison. The peak-to-peak current of the two Pt-nanoS sensors match by $80 \%$ and $75 \%$ in presence and absence of $\mathrm{H}_{2} \mathrm{O}_{2}$, respectively. The difference in the current of the two sensors could be attributed to the variability in the deposition of Pt-nanoS. The variability could be minimized by further optimizing the electrodeposition parameters such as the range of the applied voltage in LSV as well as the number of repetitions.

Alternatively, DiRIC can effectively address the Pt-nanoS 

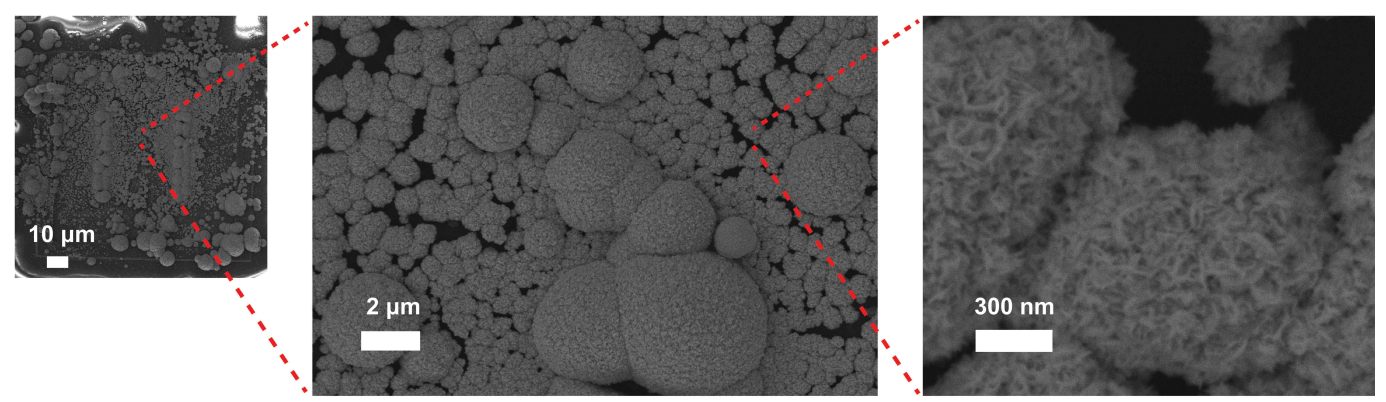

Fig. 17: SEM images at different magnification of first demonstrated Pt-nanoS grown on the top metal layer of CMOS, This is the $\mathrm{WE}_{\text {main }}$ on the same substrate as DiRIC.

TABLE V: Summary of CV measurements with on-die sensors

\begin{tabular}{cccc}
\hline \multirow{2}{*}{ Solution } & \multirow{2}{*}{ Pt-nanoS } & \multicolumn{2}{c}{ Peak-to-peak current (nA) } \\
& & $\mathrm{WE}_{\text {main }, A}$ & $\mathrm{WE}_{\text {main }, B}$ \\
\hline PBS $100 \mathrm{mM}$ & Yes & 45 & 34 \\
$\mathrm{H}_{2} \mathrm{O}_{2} 1 \mathrm{mM}$ & Yes & 142 & 114 \\
$\mathrm{PBS} 100 \mathrm{mM}$ & No & 3 & 2 \\
$\mathrm{H}_{2} \mathrm{O}_{2} 1 \mathrm{mM}$ & No & 5 & 3.5 \\
\hline
\end{tabular}

sensor variability. This includes for example sensor offsetremoval through a differential measurement. In the case of Cyclic voltammetry, The differential measurement could be performed between a Pt-nanoS electrode, $\mathrm{WE}_{\text {main }}$, and a bare $\mathrm{Al}$ electrode, $\mathrm{WE}_{b k g}$, kept in a single fluid chamber to remove a DC offset from the acquired voltammogram (See Fig. 19). A more effective offset removal, that can be applied in Chronoamperometry measurement, is through a differential measurement between two Pt-nanoS electrodes kept in two separate chambers containing two solutions: one with a known (e.g. zero) concentration of the target molecule (at $\mathrm{WE}_{b \mathrm{~kg}}$ ) and the second solution with the unknown concentration (at $\mathrm{WE}_{\text {main }}$ ).

Moreover, the variability in the sensitivity of on-die PtnanoS electrodes could be addressed through DiRIC using a two-step calibration scheme. In a first step, the sensor sensitivity is calculated through measuring the sensor current at two or more known concentrations of the target molecule; In a second step the PGA gain and the system clock frequency, $f_{c l k}$, are configured such that the sensor sensitivity multiplied by the gain of DiRIC is kept constant among all devices.

4) Discussions: The growth of Pt-nanoS on the top metal layer is demonstrated here to increase the current level of the on-die sensor by an order of magnitude. This enables the integration of miniaturised and highly sensitive redoxbased sensors with the readout systems on CMOS and further improves upon CMOS sensor arrays for the detection of metabolites with improved SNR and LOD.

A comparison with state-of-the-art is presented in Table VI showing DiRIC is the first fully-integrated readout circuit that samples and subtracts, at the same clock phase, differential sensor currents with an overall cross-scale dynamic range of $156 \mathrm{~dB}$. The presented work is also the first to demonstrate

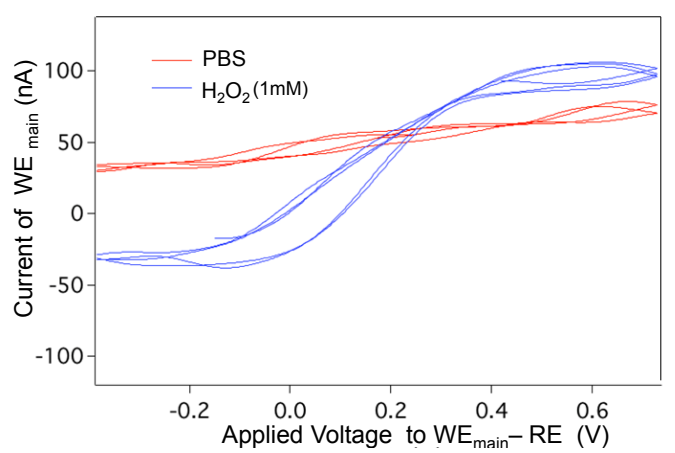

Fig. 18: The repetitive cyclic voltammograms acquired with DiRIC and on-die sensors developed with Pt-nanoS.

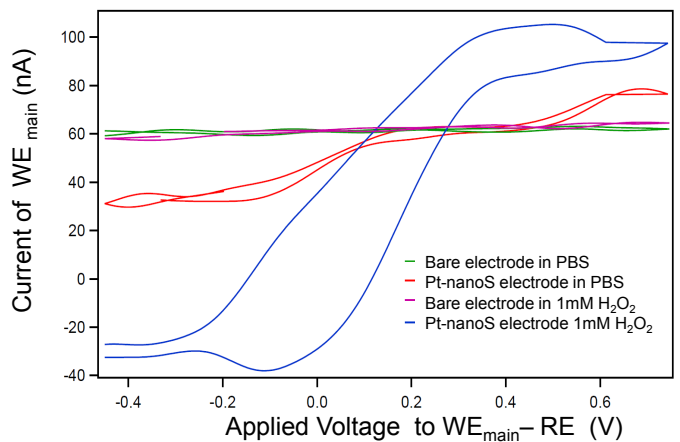

Fig. 19: Cyclic voltammograms with DiRIC and on-die sensors in presence and absence of Pt-nanoS.

the heterogeneous integration of Pt-nanoS sensors on the IC developed in CMOS technology.

\section{CONCLUSIONS}

DiRIC is presented for differential measurement of amperometric sensors using SC technology. DiRIC can measure currents within $\pm 20 \mu A$ with minimum input referred noise of $0.47 \mathrm{pA}$ and an input leakage current of $2.1 \mathrm{nA}$.

The bio-nano-sensor and DiRIC are co-designed to allow generation of the background current through $\mathrm{WE}_{b k g}$, and its subtraction from the current of $\mathrm{WE}_{\text {main }}$ through DiRIC. A method for the preparation of a differential bio-nano-sensor 
TABLE VI: Comparison summary with state-of-the-art integrated circuits for amperometric biosensors

\begin{tabular}{|c|c|c|c|c|c|c|c|c|c|}
\hline Paper & Measurement mode & Sampling of $I_{i n 1,2}$ & Min current & Max current & Cross-scale DR* & Technology & Power cons. & On-die WE & Nano type \\
\hline ISSCC'06[10] & differential & different clock phases & - & - & - & $0.5 \mu \mathrm{m}$ & - & No & - \\
\hline TBCAS'13[19] & differential & same clock phase & - & - & - & $0.18 \mu \mathrm{m}$ & $240 \mathrm{~mW}$ & No & - \\
\hline TBCAS'13[20] & single & - & $24 \mathrm{pA}$ & $0.35 \mu \mathrm{A}$ & $83 \mathrm{~dB}$ & $0.35 \mu \mathrm{m}$ & $0.19 \mathrm{~mW}$ & Yes & 3D gold \\
\hline TBCAS'16[21] & single & - & $0.5 \mu \mathrm{A}$ & $7 \mu \mathrm{A}$ & $43 \mathrm{~dB}^{* *}$ & $0.18 \mu \mathrm{m}$ & $0.07 \mathrm{~mW}$ & Yes & Carbon nanofibre \\
\hline TCAS-I'06[22] & single & - & $0.05 \mathrm{pA}$ & $\pm 0.1 \mu \mathrm{A}$ & $132 \mathrm{~dB}$ & $0.5 \mu \mathrm{m}$ & $11 \mu \mathrm{W}$ & No & - \\
\hline TBCAS'07[23] & single & - & $0.1 \mathrm{pA}$ & $\pm 0.5 \mu \mathrm{A}$ & $140 \mathrm{~dB}$ & $0.5 \mu \mathrm{m}$ & $1.27 \mathrm{~mW}$ & No & - \\
\hline TBCAS'16[12] & single & - & $0.1 \mathrm{pA}$ & $16 \mu \mathrm{A}$ & $164 \mathrm{~dB}$ & $0.5 \mu \mathrm{m}$ & $241 \mu \mathrm{W}$ & No & - \\
\hline This work & differential & same clock phase & $0.47 \mathrm{pA}$ & $\pm 20 \mu \mathrm{A}$ & $156 \mathrm{~dB}$ & $0.35 \mu \mathrm{m}$ & $9.3 \mathrm{~mW}$ & Yes & Pt nano-structures \\
\hline
\end{tabular}

is proposed. Measurements performed on the developed bionano-sensors for glucose detection show substantial reduction in the sensor offset current and the time-drift through the proposed differential sensing system.

Pt-nanoS on-die sensors were demonstrated for the first time using a simple electrodeposition technique. Measurements performed by DiRIC on on-die sensors show an order of magnitude increase in the current by the introduction of PtnanoS, as well as an increase in the sensitivity of the sensor to the $\mathrm{H}_{2} \mathrm{O}_{2}$. This allows the integration of miniaturised and highly sensitive sensors with the readout systems on CMOS for redox-based amperometric sensing of metabolites such as glucose and lactate. This further paves the way for the development of miniaturized integrated devices for point-ofcare diagnostics and the internet of medical things.

\section{ACKNOWLEDGEMENT}

The authors would like to thank Dr Salzitsa AnastasovaIvanova for taking some of the SEM images, Nishanth $\mathrm{Ku}$ lasekeram for useful discussions, and Francesca Stradolini for adjusting the Android application. The project has been funded by the Imperial College Junior Research Fellowship scheme.

\section{REFERENCES}

[1] Y. Huang, Y. Liu, B. Hassler, R. Worden, and A. Mason, "A proteinbased electrochemical biosensor array platform for integrated microsystems," Biomedical Circuits and Systems, IEEE Transactions on, vol. 7, no. 1, pp. 43-51, Feb 2013.

[2] Y. T. Liao, H. Yao, A. Lingley, B. Parviz, and B. P. Otis, "A 3uw CMOS glucose sensor for wireless contact-lens tear glucose monitoring," IEEE Journal of Solid-State Circuits, vol. 47, no. 1, pp. 335-344, Jan 2012.

[3] H. Jafari, K. Abdelhalim, L. Soleymani, E. Sargent, S. Kelley, and R. Genov, "Nanostructured CMOS wireless ultra-wideband label-free pcr-free dna analysis soc," Solid-State Circuits, IEEE Journal of, vol. 49, no. 5, pp. 1223-1241, May 2014.

[4] D. Garner, H. Bai, P. Georgiou, T. Constandinou, S. Reed, L. Shepherd, W. Wong, K. Lim, and C. Toumazou, "A multichannel DNA SoC for rapid point-of-care gene detection," in Solid-State Circuits Conference Digest of Technical Papers (ISSCC), 2010 IEEE International, Feb 2010, pp. 492-493.

[5] S. Ghoreishizadeh, C. Baj-Rossi, A. Cavallini, S. Carrara, and G. De Micheli, "An integrated control and readout circuit for implantable multi-target electrochemical biosensing," Biomedical Circuits and Systems, IEEE Transactions on, vol. PP, no. 99, pp. 1-1, 2014.

[6] I. Taurino, G. Sanzo, F. Mazzei, G. Favero, G. De Micheli, and S. Carrara, "Fast synthesis of platinum nanopetals and nanospheres for highly-sensitive non-enzymatic detection of glucose and selective sensing of ions," Scientific Reports, vol. 5, 2015.

[7] J. Wang, J. Polleux, J. Lim, and B. Dunn, "Pseudocapacitive contributions to electrochemical energy storage in TiO2 (anatase) nanoparticles," The Journal of Physical Chemistry C, vol. 111, no. 40, pp. 14925$14931,2007$.
[8] B. Bozorgzadeh and P. Mohseni, "Integrated systems for high-fidelity sensing and manipulation of brain neurochemistry," in 2016 IEEE International Symposium on Circuits and Systems (ISCAS), May 2016, pp. 1830-1833.

[9] C. I. Dorta-Quiones, X. Y. Wang, R. K. Dokania, A. Gailey, M. Lindau, and A. B. Apsel, "A wireless fscv monitoring IC with analog background subtraction and UWB telemetry," IEEE Transactions on Biomedical Circuits and Systems, vol. 10, no. 2, pp. 289-299, April 2016.

[10] M. Augustyniak, C. Paulus, R. Brederlow, N. Persike, G. Hartwich, D. Schmitt-Landsiedel, and R. Thewes, "A 24x16 CMOS-based chronocoulometric DNA microarray," in Solid-State Circuits Conference, 2006. ISSCC 2006. Digest of Technical Papers. IEEE International, Feb 2006, pp. 59-68.

[11] S. S. Ghoreishizadeh, P. Georgiou, S. Carrara, and G. D. Micheli, "An integrated platform for differential electrochemical and ISFET sensing," in 2016 IEEE International Symposium on Circuits and Systems (ISCAS), May 2016, pp. 2875-2878.

[12] H. Li, C. S. Boling, and A. J. Mason, "Cmos amperometric adc with high sensitivity, dynamic range and power efficiency for air quality monitoring," IEEE Transactions on Biomedical Circuits and Systems, vol. 10, no. 4, pp. 817-827, Aug 2016.

[13] M. Ahmadi and G. Jullien, "Current-mirror-based potentiostats for threeelectrode amperometric electrochemical sensors," Circuits and Systems I: Regular Papers, IEEE Transactions on, vol. 56, no. 7, pp. 1339-1348, July 2009.

[14] S. S. Ghoreishizadeh, I. Taurino, S. Carrara, and G. D. Micheli, "A current-mode potentiostat for multi-target detection tested with different lactate biosensors," in 2012 IEEE Biomedical Circuits and Systems Conference (BioCAS), Nov 2012, pp. 128-131.

[15] K. Martin, L. Ozcolak, Y. Lee, and G. C. Temes, "A differential switched-capacitor amplifier," IEEE Journal of Solid-State Circuits, vol. 22, pp. 104-106, 1987.

[16] J. H. Fischer, "Noise sources and calculation techniques for switched capacitor filters," IEEE Journal of Solid-State Circuits, vol. 17, no. 4, pp. 742-752, Aug 1982.

[17] F. Stradolini, S. Riario, C. Boero, C. Baj-Rossi, I. Taurino, G. Surrel, G. D. Micheli, and S. Carrara, "Wireless monitoring of endogenous and exogenous biomolecules on an android interface," IEEE Sensors Journal, vol. 16, no. 9, pp. 3163-3170, May 2016.

[18] F. Basilotta, S. Riario, F. Stradolini, I. Taurino, D. Demarchi, G. D. Micheli, and S. Carrara, "Wireless monitoring in intensive care units by a 3d-printed system with embedded electronic," in 2015 IEEE Biomedical Circuits and Systems Conference (BioCAS), Oct 2015, pp. 1-4.

[19] A. Hassibi and T. Lee, "A programmable 0.18 um CMOS electrochemical sensor microarray for biomolecular detection," Sensors Journal, IEEE, vol. 6, no. 6, pp. 1380-1388, Dec 2006.

[20] M. Nazari, H. Mazhab-Jafari, L. Leng, A. Guenther, and R. Genov, "CMOS neurotransmitter microarray: 96-channel integrated potentiostat with on-die microsensors," Biomedical Circuits and Systems, IEEE Transactions on, vol. 7, no. 3, pp. 338-348, June 2013.

[21] K. A. A. Mamun, S. K. Islam, D. K. Hensley, and N. McFarlane, "A glucose biosensor using CMOS potentiostat and vertically aligned carbon nanofibers," IEEE Transactions on Biomedical Circuits and Systems, vol. 10, no. 4, pp. 807-816, Aug 2016.

[22] A. Gore, S. Chakrabartty, S. Pal, and E. C. Alocilja, "A multichannel femtoampere-sensitivity potentiostat array for biosensing applications," IEEE Transactions on Circuits and Systems I: Regular Papers, vol. 53, no. 11, pp. 2357-2363, Nov 2006.

[23] M. Stanacevic, K. Murari, A. Rege, G. Cauwenberghs, and N. V. Thakor, "VLSI potentiostat array with oversampling gain modulation for wide-range neurotransmitter sensing," IEEE Transactions on Biomedical Circuits and Systems, vol. 1, no. 1, pp. 63-72, March 2007. 


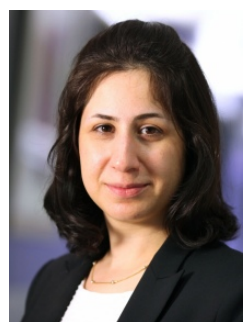

Sara Ghoreishizadeh (AM'10-M'15) received the $\mathrm{Ph} . \mathrm{D}$. degree from Ecole Polytechnique Federale de Lausanne (EPFL), Switzerland, in 2015, following the M.Sc. and B.Sc. degrees from Sharif University of Technology, Iran, in 2009 and 2007, respectively. She joined the Centre for Bio-inspired Technology (CBIT) at the Department of Electrical and Electronic Engineering, Imperial College London, in 2015 where she is currently a Junior Research Fellow. Her current research focus is integrated circuit and system design for implantable and wearable medical devices. This includes Lab-on-CMOS for metabolite sensing, biosensor and circuit co-design for enhanced detection, auto-calibration of biosensors, and power transmission and data communication in systems with multiple implants. She has been a Review Committee Member for IEEE conferences including ICECS 2016 and BioCAS 2017. She is currently on the editorial board of the Journal of Microelectronics and a member of IET, IEEE, and the IEEE Circuits and Systems (CAS) Society.

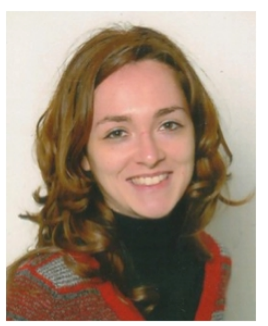

Irene Taurino received the B.Sc. and the M.Sc degree in Biomedical Engineering both cum laude from Politecnico di Torino (Italy) in 2008 and 2010, respectively. She also got the M.Sc. degree in Biomedical Engineering from Politecnico di Milano (Italy) in 2010. She carried out her PhD at EPFL on nanostructures for electrochemical biosensors. She also worked in the Integrated Systems Laboratory (LSI) as Post-Doc under the supervision of Prof. Giovanni De Micheli and Dr. Sandro Carrara.

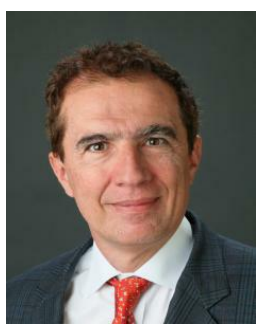

Giovanni De Micheli (F'94) is a Professor and the Director of the Institute of Electrical Engineering with EPFL, Lausanne, Switzerland. He is also the Program Leader of the Nano-Tera.ch Program. He is the author of Synthesis and Optimization of Digital Circuits (McGraw-Hill, 1994), co-author and/or coeditor of eight other books and of over 750 technical articles. His research interests include several aspects of design technologies for integrated circuits and systems, such as synthesis for emerging technologies, networks on chips, and 3-D integration. He is also interested in heterogeneous platform design, including electrical components and biosensors, and in the data processing of biomedical information. $\mathrm{He}$ is a fellow of ACM and a member of the Academia Europaea. He is member of the Scientific Advisory Board of IMEC (Leuven, B), CfAED (Dresden, D), and STMicroelectronics. Prof. De Micheli was a recipient of the 2016 IEEE/CS Harry Goode Award for seminal contributions to design and design tools of networks on chips, the 2016 EDAA Lifetime Achievement Award, the 2012 IEEE/CAS Mac Van Valkenburg Award for contributions to theory, practice, and experimentation in design methods and tools, and the 2003 IEEE Emanuel Piore Award for contributions to computer-aided synthesis of digital systems. He also received the Golden Jubilee Medal for outstanding contributions to the IEEE CAS Society in 2000, the D. Pederson Award for the best paper on the IEEE TRANSACTIONS ON CAD/ICAS in 1987, and several Best Paper Awards, including the DAC (1983 and 1993), the DATE (2005), the Nanoarch (2010 and 2012), and the Mobihealth (2016). $\mathrm{He}$ has been serving the IEEE in several capacities, namely: Division 1 Director (2008-9), co-founder and President Elect of the IEEE Council on EDA (20052007), President of the IEEE CAS Society (2003), an Editor-inChief of the IEEE TRANSACTIONS ON CAD/ICAS (19972001).of several conferences, including the Memocode (2014), the DATE (2010), the pHealth (2006), the VLSI SOC (2006), the DAC (2000), and the ICCD (1989). His citation h-index is 92 according to Google Scholar.

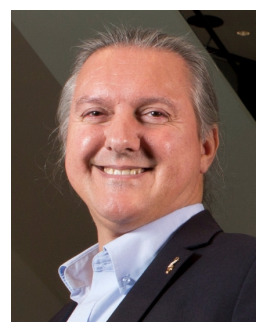

Sandro Carrara is an IEEE Fellow for his outstanding record of accomplishments in the field of design of nanoscale biological CMOS sensors. He is also the recipient of the IEEE Sensors Council Technical Achievement Award in 2016 for his leadership in the emerging area of co-design in $\mathrm{Bio} / \mathrm{Nano} / \mathrm{CMOS}$ interfaces. He is a faculty member (MER) at the EPFL in Lausanne (Switzerland). He is former professor of optical and electrical biosensors at the Department of Electrical Engineering and Biophysics (DIBE) of the University of Genoa (Italy) and former professor of nanobiotechnology at the University of Bologna (Italy). He published 7 books, one as author with Springer on Bio/CMOS interfaces and, more recently, a Handbook of Bioelectronics with Cambridge University Press. He also published more than 200 scientific papers and is author of 13 patents. He is now Associate-Editor-in-Chief of the IEEE Sensors Journal, founder and Editor-in-Chief of the journal BioNanoScience by Springer, and Associate Editor of IEEE Transactions on Biomedical Circuits and Systems. He has been appointed as IEEE Sensors Council Distinguished Lecturer for the years 2017-2019, and CASS Distinguished Lecturer for the years 2013-2014. His work received several international recognitions, including a NATO Advanced Research Award in 1996 for original contribution to the physics of single-electron conductivity in nanoparticles, seven Best paper/poster Awards in international conferences along the years. In 2014, he has been the General Chairman of the Conference IEEE BioCAS, the premier worldwide international conference in the area of circuits and systems for biomedical applications.

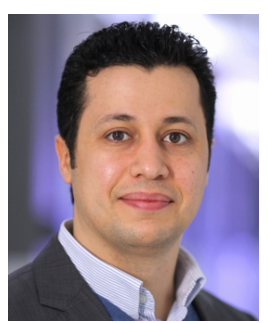

Pantelis Georgiou (AM'05 M'08 SM'13) received the M.Eng. degree in electrical and electronic engineering and the $\mathrm{Ph} . \mathrm{D}$. degree from Imperial College London (ICL), London, U.K., in 2004 and 2008, respectively. He is currently a Reader with the Department of Electrical and Electronic Engineering, ICL, where he is also the Head of the BioInspired Metabolic Technology Laboratory, Centre for Bio-Inspired Technology. His research includes bio-inspired circuits and systems, CMOS based Labon-Chip technologies, and application of microelectronic technology to create novel medical devices. He has made significant contributions to integrated chemical-sensing systems in CMOS, conducting pioneering work on the development of ISFET sensors, which has enabled applications, such as point-of-care diagnostics and semiconductor genetic sequencing and has also developed the first bio-inspired artificial pancreas for treatment of Type I diabetes using the silicon-beta cell. He received the IET Mike Sergeant Medal of Outstanding Contribution to Engineering in 2013. He is a member of the IET and serves on the BioCAS and Sensory Systems technical committees of the IEEE CAS Society. He is also the CAS representative on the IEEE Sensors council and the IEEE Distinguished Lecturer in Circuits and Systems. 